\title{
CITIZEN PARTICIPATION \& DIGITAL TOOLS TO IMPROVE PEDESTRIAN MOBILITY IN CITIES
}

\author{
O. Ertz ${ }^{1, *}$, A. Fischer ${ }^{2}$, H. Ghorbel ${ }^{3}$, O. Hüsser ${ }^{3}$, R. Sandoz ${ }^{1}$, A. Scius-Bertrand ${ }^{2}$
}

(1) HEIG-VD, MEI - Media Engineering Institute, Yverdon-les-Bains, Switzerland (olivier.ertz@heig-vd.ch, romain.sandoz@heig-vd.ch) (2) HEIA-FR, iCoSys - Institute of Complex Systems, Fribourg, Switzerland (andreas.fischer@hefr.ch, anna.scius-bertrand@hefr.ch ) (3) HE-Arc, Data Analytics Group, Neuchâtel, Switzerland (hatem.ghorbel@he-arc.ch, olivier.husser@he-arc.ch )

KEY WORDS: walkability, mobility of seniors, citizen engagement, crowdsourcing, route personalization, smart citizen.

\begin{abstract}
:
In this work, we present a framework supported by mobile and web apps and able to propose personalized pedestrian routes that match user mobility profile considering mobility impediments factors. We explain how these later have been defined using a pedestriancentric approach based on travel experiences as perceived in the field by senior citizens. Through workshops, six main factors that may influence pedestrian route choices were revealed: passability, obstacle in path, surface problem, security, sidewalk width, slope. These categories were used to build digital tools and guide a citizen participatory approach to collect geolocated points of obstacle documented with walkability information (picture, category, impact score, free comment). We also involved citizens to evaluate these information and especially senior referents for validation. Finally we present how we connect these points of obstacle with a pedestrian network based on OpenStreetMap to configure a routing cost function. The framework has been partially deployed in 2020 with limited people due to the pandemic. Nonetheless, we share lessons learned from interaction with citizens in the design of such a framework whose underlying workflow is reproducible. We plan to further assess its relevance and sustainability in the future.
\end{abstract}

\section{INTRODUCTION}

\subsection{Context and problem statement}

The average age of people experiencing discomfort in moving around is 69 years, with a very noticeable increase from $79-80$ years of age (Hauet and Ravaud, 2002). The unevenness of sidewalks, holes, obstacles (Chaudet, 2012), the absence of benches for resting (Huguenin-Richard, 2014) and the inadequacy of lighting (Chapon and Renard, 2009) constitute obstacles to regular walking. Yet, this mode of travel is largely predominant from the age of 70 (Dumas, 2012) and it is essential to the health maintenance of seniors living at home. In order to promote the mobility of senior citizens, it therefore seems necessary to consider actions to make pedestrian travel safer and easier through the appropriate design of the urban environment (Dumas, 2012). While cities of Aarau, Bâle, Bellinzone, Coire and Neuchâtel have been awarded, given a recent study (Killer et al., 2020), cities in Switzerland still have significant potential for improvement. We must also highlight the fact that sidewalks are increasingly becoming an area of interest in the era of new mobilities and new functionalities linked to the smart city (Baraud-Serfaty, 2020).

Because there is no reason that, as you age and/or have specific mobility constraints, your range of pedestrian mobility should be reduced due to obstacles which blocks or impedes your travel: what if you were unlucky enough to be convalescing on crutches to get to an appointment in Neuchâtel, a city whose pedestrian network you don't really know much about? what if you managed to convince your grandmother, who has become fearful with age, to walk again on the sidewalks of her city? what if you were in a wheelchair and want to visit Bern, which you only knew before a sad accident? what if you were lucky enough to be driving a stroller to make your child discover the city and its inhabitants?

Considering so many different situations in life that could appreciate pedestrian mobility assistance, this paper presents our contribution which aims to help navigation in a city by visualizing current obstacles on a map and to personalize routes according to user profiles and preferences. In other words, digital tools to help in bypassing the obstacles while waiting for them to be solved by city planners. Such a problem statement makes it necessary to challenge several issues inherent to urban walkability under constraints. Therefore, we propose a framework supported by mobile and web apps based on known tools, algorithms and methods in order to define a sustainable and reusable workflow to assess obstacles in urban context, weight their impact and as a result propose personalized route calculation that match user mobility profile. It is built of:

1) pedestrian-centric approach to define an adequate typology of pedestrian mobility impediments by making concrete experiences as perceived in the field by senior citizens;

2) citizen participatory approach for in situ and remote crowdsourcing to collect geolocated points of obstacle documented with walkability information;

3) evaluation by citizens of walkability information per type of mobility impairments and validation by senior referents;

4) creation of a geospatial database to build and store the network of pedestrian ways using OpenStreetMap and the layer of the collected/documented points of obstacle;

5) weighting of each street segment by merging/snapping the layer of points of obstacle with the pedestrian network;

6) personalized route calculation using these weights to calculate a shortest path considering user preferences.

This work is known as thec modos project at the University of Applied Sciences and Arts of Western Switzerland (HES-SO) which is the funding body. Members of the research team have already carried out works related to the urban pedestrian mobility topic, notably (Liu et al., 2017) and (Blanc et al., 2019).

\subsection{Related work}

Below we present background of other related works on the urban pedestrian mobility topic carried out by research teams about

\footnotetext{
${ }^{*}$ Corresponding author. E-mail address: oliver.ertz@heig-vd.ch (O. Ertz).
} 
obstacles typology, collecting point of obstacles in the urban field, building pedestrian ways (esp. sidewalks) to be connected together to form a graph, and route personalization techniques.

Typology of obstacles: as mentioned above, the urban infrastructure has many characteristics perceived as impediments undermining pedestrian mobility. In the city of Alghero (Italy) a contingent field survey has been conducted (Blečić et al., 2016) to clarify variables associated with the general perception of walkability which can be manifold (useful sidewalk width; architectural, urban and environmental attractions; density of shops, bars, services, economic activities; vehicles-pedestrians separation; cyclability; opportunities to sit; shelters and shades; car roadway width; street lighting). In the city of Washington (United States), Project Sidewalk, has a strong concern in supporting population with ambulatory disabilities (Saha et al., 2019) and five high-priority areas that impact walkability have been identified, mainly drawn from Americans with Disabilities Act standards (curb ramps, missing curb ramps, sidewalk obstacles, surface problems, and the lack of a sidewalk on a pedestrian pathway). In other words, the latter does focus on the design of the pedestrian infrastructure. In Yverdon-les-Bains (Switzerland), with a similar focus, the modos project does call upon senior citizens as referrers of a particular awareness to help in making concrete the concept of obstacle.

Collecting obstacles: with the rise of digital tools, new methods to collect data on street-level appeared: in situ crowdsourcing with users capturing data in the field, automatic data capture using sensors, and remote crowdsourcing using streetscape imagery. While Project Sidewalk (Saha et al., 2019) falls into the 3rd category, theirs authors discuss the benefits and drawbacks of these approaches that should be considered complementary. For the modos project, we combine in situ and remote crowdsourcing, however by engaging local communities and adopting a pedestrian-centric approach similar to the project CAST which underlines the ability of a citizen-centered participatory process including ICT tools to enable the involvement of a large number of citizens (Carbone et al., 2018).

Pedestrian network creation: in terms of mapping practices for pedestrian navigation, route personalization requires ways to be connected together and form a graph, in our case, ways along which pedestrians are experiencing their mobility. The OpenSideWalks project does promote the use of OpenStreetMap as the repository for collection of walkability information in a standardized manner. It provides a workflow ("OpenSidewalks Editing Tutorial," n.d.) to create the fundamental pedestrian network layer using OSM-specific tagging for each pedestriancentric feature. For the modos project, while we adopted part of this workflow (especially to ensure the topology of the pedestrian network, notably by the mapping of sidewalks), we also added a specific layer of points of obstacle.

Route personalization: to personalize a route, segments of a network have to be weighted based on some factors and then integrated into a routing cost function. That's what Novack et al. (2018) are doing by considering four factors (street length, greenness, sociability, and quietness). These factors are strictly extracted from other related data from OpenStreetMap, even the sociability of streets which seems difficult to measure and which should perhaps be better appreciated by the citizens themselves. For the modos project, while we use a similar cost function based on the length as well as weighted factors and user-defined preferences, we do make the weighted factors objective on the basis of citizens' experiences.

\section{METHODOLOGY}

To personalize a route according to user's mobility profile, we have chosen to calculate the route according to the weighting of obstacles associated with the user profile. Two options are then available: exclude categories of obstacles (e.g. slopes) or choose a route that will be calculated according to the profile. To achieve this result, several steps are necessary: defining obstacle categories, collecting obstacle geolocation data, user engagement, labelling obstacles and then assigning them a difficulty level according to different mobility profiles.

\subsection{Toward a typology driven by seniors}

To be able to map and document obstacles to pedestrian mobility, we must define a typology of those. Our approach was to include end-users in the process for our typology to reflect their reality, making concrete experiences as perceived in the field by senior citizens. Therefore we worked with volunteers of the senior citizens' council of the city of Yverdon-les-Bains (COSY) during two workshops. The first workshop aimed to identify and assess obstacles found in the old town of Yverdon-les-bains. To do so, we defined three different routes which were explored by elderresearcher pairs. Along the way, the volunteers notified the researcher of each elements they considered as an obstacle and why. During the second workshop, we discussed around the collected obstacles with the same group of volunteers from the COSY. They were split in two groups to discuss, classify, and rate (on a scale from 1 to 5) the impact of each obstacles. The expected result of such workshops is to get a typology of obstacles to be used to develop our digital tools.

\subsection{Data acquisition}

To weight segments of the pedestrian network we use an intermediate data layer. It stores walkability information about points of obstacle collected along the network with the essential element of a street-level picture of each obstacle. To collect these pictures we first used a mass-capture approach with two different solutions: (1) the CityFeel backpack which integrates multiple sensors including geo-positioning with a hemispheric camera (Gallinelli et al., 2017), (2) a simpler solution based on a GoPro camera with GPS attached on a scooter. But any geolocated image can be used to feed our framework, as long as it is a pedestrian view (versus roadway view, e.g. Google Street View).

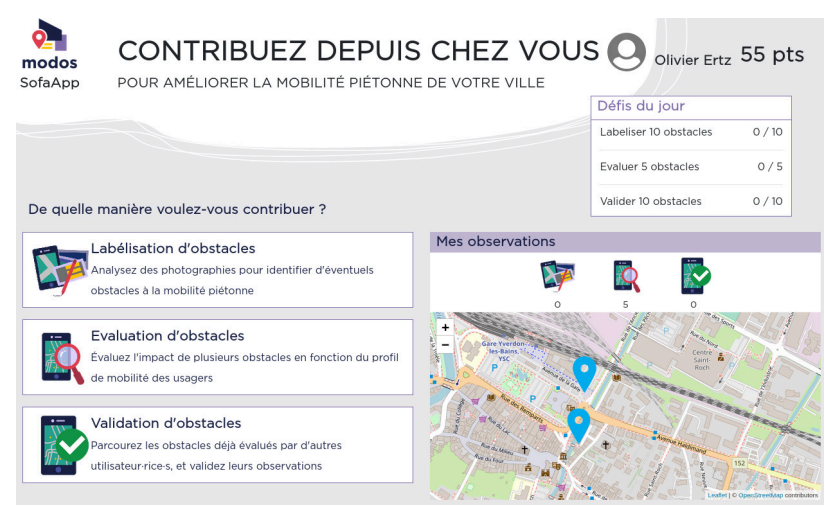

Figure 1. Start page of the sofa web app allowing to perform labelling, evaluation of impact and validation.

Then we need inputs from citizens to document these points of obstacle with walkability information: a classification (based on the typology) and the evaluation/validation of their impact. To collect them, we developed two crowdsourcing apps to be used remotely (Figure 1) and in situ (Figure 2): 
- $\quad$ sofa app: a web app which allows to manually classify images, evaluate/validate impact (see Labelling section)

- explorer app: a mobile app to take pictures of obstacles in the field (in addition to the mass-capture) and allowing to directly classify and evaluate impact.

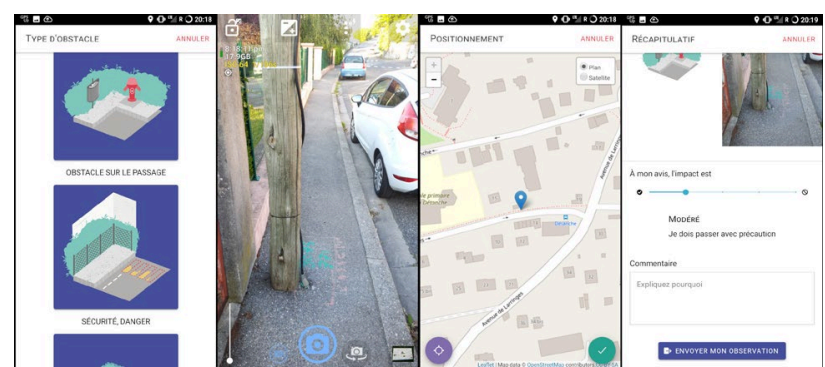

Figure 2. Main screens of the explorer mobile app

These apps do also collect the mobility profile of each citizen contributor. It is of importance to put contributions into perspective for the sake of route personalization. The purpose of these two apps is to support the citizen participatory approach providing a sense of belonging, especially by organizing events during which volunteers gather to use the applications.

\subsection{User engagement}

To ensure data quality and quantity, our citizen participatory approach requires strong user engagement. Thus, we firstly define the tasks we need the volunteers to fulfil : (a) take pictures of obstacles, (b) categorize pictures of obstacles and evaluate categorizations, (c) evaluate the impact of the obstacles, (d) validate the impact of the obstacles, (e) evaluate the relevancy of personalized routes. Then we classify these tasks (Table 1) considering Geiger and Shader (2014) crowdsourcing systems.

\begin{tabular}{|c|l|l|}
\hline non-emergent & task (a) & task (e) \\
\hline emergent & task (b) & tasks (c), (d), (e) \\
\cline { 2 - 3 } & homogeneous & heterogeneous \\
\cline { 2 - 3 }
\end{tabular}

Table 1. emergent: the value is derived from the entirety of contributions / non-emergent: it is derived directly from each contribution / homogeneous: contributions are seen as qualitatively identical / heterogeneous: contributions are valued differently according to their individual qualities.

Based on this classification, we note that most of the tasks asked to volunteers are emergent and heterogeneous. This implies that the overall value of the contributions is derived from the entirety of the contributions and each contribution does not have the same individual value. It confirms that the contributions must be rated, sorted, and evaluated in a process involving multiple human inputs. This is what we tend to achieve with the support of the crowdsourcing web and mobile apps, especially the so-called sofa app. It does also emphasize the subjective aspect of obstacles classification and evaluation.

Moreover, it highlights the fact that the tasks to fulfil require a significant thought process for contributions to be valuable. As tasks like taking a picture of an obstacle (crowdprocessing) requires low expertise, most of our other tasks (mainly crowdsolving) requires more reasoning and a certain level of knowledge. This underlines the importance to find ways to motivate users and to give time and means to fulfil those tasks.

To address these motivational aspects, we think relevant to consider recommendations proposed by Lotfian et al. (2020) :
Sensitization and learning:

- provide informative material on the project to allow volunteers to learn new things and find meaning in their tasks;

- provide updates to volunteers about their contributions and the results of their work;

- include people who are not directly impacted by the problematic of mobility impairment, but willing to help for the greater good, and generate intergenerational support.

Social aspect:

- provide the opportunity for volunteers to meet with experts by organizing workshops (e.g. in the field mapping party);

- build a community of volunteers, by giving the possibility of social interactions, digitally or in real life;

- Approach existing communities which are concerned by the project topics (councils, associations, authorities).

\subsection{Labelling}

Images from the scooter were not labelled with a category of obstacle. Moreover, given the mode of capture, the majority of the pictures does even not contain obstacles. Therefore, the large number of pictures was classified automatically using a machine learning algorithm to keep only those that have a high probability of "containing an obstacle" and to set a first category assigned (see next section).

A manual step is used to correct potential auto-labelling errors using the sofa app. The latter has three features: labelling, evaluation and validation. By means of labelling, the user can select all the images belonging to a category (previously chosen by the system or by the user) using a similar interface as for image-recognition based CAPTCHA, i.e. displaying and labelling several images at once. Pictures from the scooter are displayed in priority, but some data from the explorer app can also be shown to the user.

For the evaluation feature, an obstacle is displayed as well as the label (category) that has been assigned to it (automatically or manually). If the user does not agree with this label he or she has the possibility to choose another label. If the label was proposed automatically then the label assigned by the user will be kept, if the labels were assigned by human users then the label with the most votes is chosen. In the case of a tie, the label assigned via the explorer app is chosen or by default the last label.

\subsection{Weighting}

Setting a difficulty level (i.e. impact weight on walkability) of an obstacle takes place in two steps: (1) assigning a difficulty level by any lay contributor, e.g. students who try to put themselves into the position of a senior, (2) validating the results by experts, i.e. seniors with the corresponding mobility profile.

During the evaluation, users are invited to evaluate the difficulty of an obstacle according to four mobility profiles. The evaluation is done via a scale from 0 to 5 ( 0 Non-existent: I can pass without difficulty; 1 Low: I can still pass; 2 Moderate: I must pass with caution; 3 Marked: I have difficulty passing; 4 Severe: I have great difficulty passing; 5 Blocking: I can't get through at all). Here the user must put himself in the shoes of the different mobility profiles. This choice was made because it was simpler to acquire this data in an age range below 65 years.

The validation part was developed to ensure the quality of the results of the evaluation, which was done mostly by people who are not senior and/or who do not have mobility difficulties. Only seniors and people with limited mobility can access this part. 
According to their mobility, users can confirm or not the level of difficulty assigned to an obstacle.

\subsection{Routing}

Once the obstacles have been assigned a label and a level of difficulty (i.e. impact) according to a mobility profile, routing is performed using the shortest path taking into account specific weights for the obstacles according to the profile. For each segment of the network, the related point of obstacle with the highest difficulty level is selected. Then the weight of a segment is calculated as follows: the size of the segment in meters multiplied by a ratio determined according to the difficulty level of the segment. Two types of weight scales have been designed: one to determine a fast route trying to avoid the most constraining obstacles and another one for an easy route avoiding as much as possible any difficulty. Weights for obstacles 1-5 (according to the scale defined above) are as follows:

- fast route : $[1,5,20,100,100000]$; - easy route : $[200,300,500,750,100000]$.

For an obstacle, for which there is not yet an evaluation for a specific profile, the weight entered through the explorer app application is used. As a result, using these weights the shortest path calculation is personalized according to the mobility profile.

\section{EXPERIMENTATION \& RESULTS}

\subsection{Getting the typology of obstacles}

To begin we found good references from other similar projects such as Project Sidewalk to predefine an early typology. We had then to test and determine if it was reflecting the reality of our senior referents. Indeed, the intent is to adopt a pedestrian-centric approach to define an adequate typology of pedestrian mobility impediments by making concrete experiences as perceived in the field by senior citizens. So we conducted a pedestrian exploration, called urban safari, and a categorization workshop.

\subsubsection{Urban safari}

The design of the pedestrian exploration began by analysing the streets that are the most used by the pedestrian in Yverdon-lesBains down town. We decided to go through three main locations in the city centre. During the exploration, when a senior volunteer felt that something should be considered an obstacle or a difficulty, we recorded a new diagnosis element by taking pictures and notes according to a dedicated form: physical characteristic of the obstacle, category using the early typology, notes about the participant's feeling about it. Finally we had gathered 134 pictures representing 76 unique obstacles (an obstacle can be captured from different points of view, hence the higher number of pictures than obstacles). Due to some undocumented pictures, irrelevant obstacles or the lack of tangible physical evidence of obstacle, some of the pictures were discarded to end up with 70 unique obstacles. We finally decimated the remaining 70 obstacles identified as such by eliminating duplicates due to some overlaps in the chosen paths (two different groups of participants may have taken pictures of the same obstacle), hence keeping only 57 pictures.

\subsubsection{Workshop}

The urban safari allowed us to gather obstacles from the field with the point of view of senior citizens. The goal of the workshop is to define their typology matching their perspective. Eight senior citizens were present (Figure 3). After a short briefing, we randomly split participants in two groups not allowed to communicate with each other. Each had 20 minutes to discuss and distribute the 57 selected printed pictures into typological clusters according to unique categories they defined with their own words. This phase resulted in the following typology which we refined and used all along the project :

- Passability: related to some stepping over (access to sidewalk without curb ramp, high kerbside, small stairs, ...)

- Obstacle in the path: permanent or temporary (e.g. lamp pole, fire hydrant, restaurant easel, ...)

- Security: related to the traffic, missing or inadequate urban design (e.g. missing signs, crossing, visibility, island, ...)

- Slope: steepness of the street (e.g. may also be critical in unfavourable weather conditions)

- Surface problem: which impedes progress when rolling (e.g. wheel walker, wheelchair) or is likely to cause a fall (stumble)

- Sidewalk width: too narrow to cross other people or to pass with walking aid

- Bonus: any positive asset seen as beneficial

In a second step, volunteers had to give a score between 1 and 5 ( 1 being the less obstructing and 5 the most) to each category according to its perceived impact on walkability. We noticed differences of opinion, what was expected because each participant does not necessarily have the same walking difficulties. Group A evaluated the difficulty of the Security category significantly higher (4.0) than group B (2.0). Also, surface problem seemed to have the highest impact (5.0) for group A as it has been rated lower by group B (3.9). Very few pictures were categorized as bonus but the discussion between the participant highlighted that the ability to find beneficial amenities - such as public bench, shaded areas, parks, ... - may be decisive when planning a walk. This category has been further discarded from our terminology so as to focus on the negative assets. Nonetheless, we added the special category other that can also be used for positive assets if the volunteer feels the need to record such information (may be used as an additional layer to enhance route personalization).

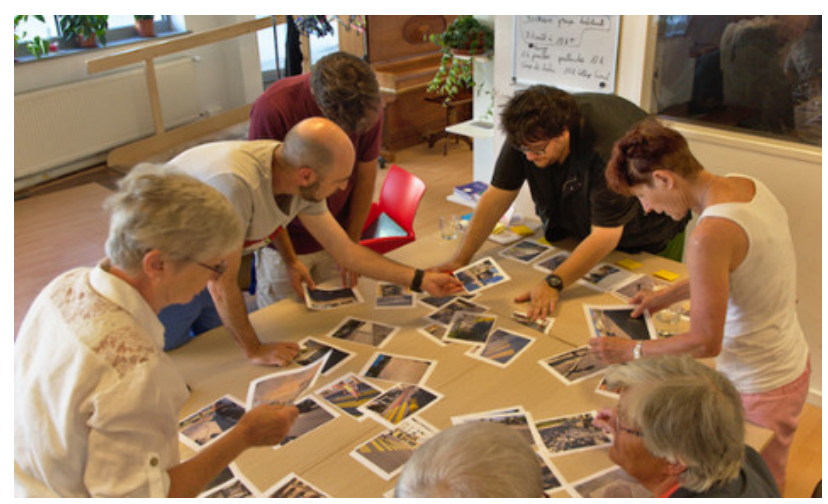

Figure 3. Workshop with senior citizens from COSY

\subsection{Data acquisition}

\subsubsection{Mass-capture}

From our experience, the CityFeel backpack has the advantage of high-accuracy geo-positioning but generates images that are not adapted because of hemispherical pictures that have to be postprocessed in order to be used (especially for the machine learning that needs pictures that look like what a citizen might capture in the field as ground truth). The GoPro solution was on the other hand a low-cost and good way to acquire a good amount of pictures without too much effort (800 images / hour for a path of $10 \mathrm{~km})$ with usable pictures.

\subsubsection{Mapping party}

With the support of the dedicated explorer app we organized a mapping party with 12 volunteers. 6 volunteers were members of 
the project and 6 were people outside the project with little to no knowledge about the project. The goals of this mapping party were: evaluate technical efficiency and stability, evaluate the application usability, determine the motivations of the participants. This event was segmented in four phases : (1) recruiting, (2) briefing, (3) mapping, (4) debriefing.

When recruiting our volunteers, we provided them with general information about the project. We sent them a user guide of the application with a help memo about the typology of obstacles they will have to use. We then invited them to a videoconference (due to the pandemic) to present them the project a bit more in depth and gave them the necessary instructions for the day of the mapping party.

During the mapping party, participants were assigned randomly to one of the three predefined routes of $2 \mathrm{~km}$ across the city. They were asked to walk along those at their own pace to potentially find anything they may consider an obstacle. After 30 minutes of exploration, the volunteers and the team gathered to debrief and talk about their experience. As a result, the volunteers collected 215 labelled pictures, with an average of 17 per participant and a max-min of 31-5.

During the debriefing, the results of volunteers work were presented through statistics and a map showing all the collected obstacles. We then gathered their feedbacks about usability of the app, their field experience, and their motivations. Two methods were used to collect feedback: open discussion \& asynchronous individual questionnaire. Overall, the application was qualified as very easy to use and no participant reported immediate bugs within the app. However, we noticed afterwards that the geolocation of some obstacles was erroneous. Firstly, some obstacles were located several meters off their real location. Secondly, the geolocation of all the obstacles collected by one only participant were all placed at a same location. Those issues were probably due to hardware and signal limitation from the device, especially when taking pictures in narrow streets. And due to the heterogeneous type of devices, it has yet to be determined if it is also a software issue. The discussion highlighted the fact that it's hard for some of the participants to know which posture to adopt. Some started their exploration paying attention to every details of the urban architecture and tried to empathize with mobility impaired people. Making it hard for them to know which obstacles are relevant or not. The fact that we let them free to consider any obstacle without any further indication gave them a high cognitive load during the exploration. As a consequence, most of them have felt their attention lowered throughout the exploration. This issue could be addressed by attributing one specific category of obstacle to collect, or by improving the user experience design of the app by charging the user with missions (e.g. Try to identify 5 surface problem in the area). On the other hand, other volunteers did not try to empathize at all and only collected significant obstacles for their own mobility. Also, some participants felt the need to use two categories for one obstacle. That point emphasizes the subjective aspect of the data qualification and the importance of having as many inputs as possible from different users and mobility profiles in order to refine route personalization.

Finally, regarding the experience and motivation of the volunteers, a majority of the participants (9 out of the 12) stated that being able to exchange with other contributors is essential for them to feel implied in the project. Furthermore, the volunteers admitted unanimously that participating in the project made them more sensitive of the problematic and able to empathize more with mobility impaired people. Half the participants (6) felt a sense of accomplishment from the start of their exploration. Others had this feeling during the debriefing. The main incentives to participate to the project were: "Self-help, knowing that it will help other individuals and bring meaningful community changes", "The conviviality and the questions raised by the problematic". Getting to know a person directly impacted by the underlying problematic of pedestrian mobility was also mentioned as a strong motivational lever. This tends to invite us to pay attention to stimulating intergenerational help as an important factor to gather and retain volunteers.

\subsection{Machine Learning}

In order to automatically label the images collected with the scooter, a MobileNetV2 model (Sandler et al., 2018) was trained on 9976 labelled images during 1000 epochs. The model was chosen with respect to its excellent performance on the ImageNet benchmark, while having a relatively low number of weights. $70 \%$ of the data was used as training set and $30 \%$ as test set. However the dataset is very unbalanced, the no obstacle category represents $73 \%$ of the images. The accuracy for all classes reaches $88 \%$ which seems to be a good score. In reality the network detects very well the class without obstacle but very badly the class like obstacle in path and slope with respectively a F1-score of 0.55 and 0.4 . This is why we used a double model. The first one (Table 2) aims to detect if an obstacle is present or not and a second one (Table 3 ) to recognize the obstacle class.

\begin{tabular}{|l|l|l|l|l|}
\hline & Precision & Recall & F1-score & Support \\
\hline Not present & 0.86 & 0.70 & 0.77 & 341 \\
\hline Present & 0.79 & 0.91 & 0.85 & 427 \\
\hline Accuracy & & & $\mathbf{0 . 8 2}$ & $\mathbf{7 6 8}$ \\
\hline Macro avg & 0.83 & 0.80 & 0.81 & 768 \\
\hline Weighted avg & 0.82 & 0.82 & 0.81 & 768 \\
\hline
\end{tabular}

Table 2. 1st model with an accuracy for both classes of $82 \%$

\begin{tabular}{|l|l|l|l|l|}
\hline & Precision & Recall & F1-score & Support \\
\hline 1 Curb Ramp & 0.97 & 0.88 & 0.92 & 76 \\
\hline 2 Obstacle & 0.97 & 0.75 & 0.85 & 44 \\
\hline 3 Surface & 0.92 & 0.91 & 0.92 & 257 \\
\hline 5 Width & 0.82 & 0.91 & 0.86 & 108 \\
\hline 6 Security & 0.88 & 0.94 & 0.91 & 235 \\
\hline 7 Slope & 0.91 & 0.78 & 0.84 & 41 \\
\hline 8 Other & 1.00 & 1.00 & 1.00 & 7 \\
\hline Accuracy & \multicolumn{5}{|l}{} & $\mathbf{0 . 9 0}$ & $\mathbf{7 6 8}$ \\
\hline Macro avg & 0.93 & 0.88 & 0.90 & 768 \\
\hline Weighted avg & 0.90 & 0.90 & 0.90 & 768 \\
\hline
\end{tabular}

Table 3. 2nd model reaches a performance of $90 \%$ accuracy

\section{CONCLUSION}

We have presented a framework based on concrete experiences as perceived by senior citizens for personalising pedestrian routes according to different mobility profiles. The proposed methodology includes a typology of obstacles, data acquisition, labelling and weighting of obstacles applying a citizen participatory approach supported by two crowdsourcing apps.

Current technical limitations include the relatively low precision when snapping a point of obstacle to the nearest segment of the pedestrian network (e.g. snapping to the wrong side of a sidewalk). This may be addressed by adding to the explorer app a screen to validate/adjust the geolocation on a map. Another possibility would be to use drones, which may serve to regularly obtain a complete and precise picture of the pedestrian network 
of a city. The modos project has focused on the technical and functional aspects of the personalisation and the definition of a reusable and sustainable framework. In future work, we aim to reduce cognitive load, improve usability and enhance user engagement by improving overall user experience by means of $\mathrm{UX} / \mathrm{UI}$ design and gamification mechanisms. Also, a possible extension of the presented personalisation framework would be to include not only negative assets (i.e. mobility impediments factors), but also positive assets (e.g. benches, shaded areas, parks, water fountain, etc). They might be integrated into the weighting of the segments or be used as filters when choosing among different routes. Also about typology, the ability to mention that an obstacle seems temporary may be useful to organise database updates (e.g. defining a mission statement "Check if this obstacle is still relevant").

Finally, walkability information gathered by such a framework may be of value in helping city planners to solve "hotspots of poor walkability". Indeed, we expect to involve them actively in another project in preparation to develop a negotiation process between citizens and authorities.

\section{ACKNOWLEDGEMENTS}

To the volunteers of the senior citizens' council of the city of Yverdon-les-Bains (Switzerland) and David Campisi from the senior-lab.ch for his impressive leadership to engage seniors for the participatory process which is central in this project.

\section{REFERENCES}

Baraud-Serfaty, I., 2020. Le trottoir, nouvel actif stratégique. Futuribles $N^{\circ} 436,87-104$.

Blanc, N., Liu, Z., Ertz, O., Rojas, D., Sandoz, R., Sokhn, M., Ingensand, J., Loubier, J.-C., 2019. Building a Crowdsourcing based Disabled Pedestrian Level of Service routing application using Computer Vision and Machine Learning, in: 2019 16th IEEE Annual Consumer Communications \& Networking Conference. 16th IEEE Annual Consumer Communications \& Networking Conference (CCNC), IEEE, Las Vegas, NV, USA, pp. 1-5. https://doi.org/10.1109/CCNC.2019.8651850

Blečić, I., Canu, D., Cecchini, A., Congiu, T., Fancello, G., 2016. Factors of Perceived Walkability: A Pilot Empirical Study, in: Gervasi, O., Murgante, B., Misra, S., Rocha, A.M.A.C., Torre, C.M., Taniar, D., Apduhan, B.O., Stankova, E., Wang, S. (Eds.), Computational Science and Its Applications -- ICCSA 2016 , Lecture Notes in Computer Science. Springer Publishing, Cham, pp. 125-137. https://doi.org/10.1007/978-3-319-42089-9_9

Carbone, R., Saganeiti, L., Scorza, F., Murgante, B., 2018. Increasing the Walkability Level Through a Participation Process, in: Gervasi, O., Murgante, B., Misra, S., Stankova, E., Torre, C.M., Rocha, A.M.A.C., Taniar, D., Apduhan, B.O., Tarantino, E., Ryu, Y. (Eds.), Computational Science and Its Applications - ICCSA 2018. Springer Publishing, Cham, pp. 113-124. https://doi.org/10.1007/978-3-319-95174-4_9

Chapon, P-M., Renard, F., 2009. Innover dans l'habitat et l'hébergement des personnes âgées, Salon des Maires et des Collectivités Locales (SMCL), Paris Porte de Versailles.

Chaudet, B., 2012. Les territoires du Bien vieillir au prisme de la mobilité quotidienne des personnes âgées, Viriot Durandal J-P., Pihet C., Chapon P-M. (Dir) Les défis territoriaux face au vieillissement ed. La documentation Française, pp.17-30.
Dumas, C., 2012. Mobilité des personnes âgées. Gérontologie et société $n^{\circ} 141$, p.63-76.

Gallinelli, P., Camponovo, R., Guillot, V., 2017. CityFeel - micro climate monitoring for climate mitigation and urban design. Energy Procedia, CISBAT 2017 International Conference Future Buildings \& Districts - Energy Efficiency from Nano to Urban Scale 122, 391-396. https://doi.org/10.1016/j.egypro.2017.07.427

Geiger, D., Schader, M., 2014. Personalized Task Recommendation in Crowdsourcing Information Systems Current State of the Art. Decision Support Systems 65. https://doi.org/10.1016/j.dss.2014.05.007

Hauet, E., Ravaud, J-F., 2002. Handicap, gênes ou difficultés ressentis par les personnes dans le cadre de leurs déplacements en dehors du domicile - Rapport final du projet APF 00-05, Association des Paralysés de France.

Huguenin-Richard, F., 2014. La marche à pied pour les séniors : un mode de déplacement durable ? Séminaire "Vulnérabilité et mobilité", Ministère de l'écologie, du développement durable et de l'énergie, Paris, La Défense, France.

Killer, V., Hobi, S., Schweizer, T., Zweibrücken, K., 2020. Marchabilité : les villes ont encore un important potentiel d'amélioration. actif-trafiC, Mobilité piétonne Suisse, HES sciences techniques Rapperswil.

Liu, Z., Glassey Balet, N., Sokhn, M., De Gaspari, E. (Eds.), 2017. Crowdsourcing-based mobile application for wheelchair accessibility. Proceedings of the 32nd Annual International Technology and Persons with Disabilities Conference Scientific/Research.

Lotfian, M., Ingensand, J., Brovelli, M.A., 2020. A Framework for Classifying Participant Motivation that Considers the Typology of Citizen Science Projects. ISPRS International Journal of Geo-Information 9, 704. https://doi.org/10.3390/ijgi9120704

Novack, T., Wang, Z., Zipf, A., 2018. A System for Generating Customized Pleasant Pedestrian Routes Based on OpenStreetMap Data. Sensors 18, 3794. https://doi.org/10.3390/s18113794

OpenSidewalks Editing Tutorial [WWW Document], n.d. URL https://www.opensidewalks.com/files/01_OpenSidewalksEditin g.pdf (accessed 6.16.21).

Saha, M., Saugstad, M., Maddali, H.T., Zeng, A., Holland, R., Bower, S., Dash, A., Chen, S., Li, A., Hara, K., Froehlich, J., 2019. Project Sidewalk: A Web-based Crowdsourcing Tool for Collecting Sidewalk Accessibility Data At Scale, in: Proceedings of the 2019 CHI Conference on Human Factors in Computing Systems. Presented at the CHI '19: CHI Conference on Human Factors in Computing Systems, ACM, Glasgow Scotland Uk, pp. 1-14. https://doi.org/10.1145/3290605.3300292

Sandler, M., Howard, A., Zhu, M., Zhmoginov, A., Chen, L., 2018. "MobileNetV2: Inverted Residuals and Linear Bottlenecks," IEEE/CVF Conference on Computer Vision and Pattern Recognition, 2018, pp. 4510-4520, doi: 10.1109/CVPR.2018.00474 